\title{
Low Temperature Wurtz-Type Polymerization of Substituted Dichlorosilanes
}

\author{
R. D. Miller, E. J. Ginsburg, and D. Thompson \\ IBM Research Division, Almaden Research Center, 650 Harry Road, \\ San Jose, California 95120-6099, CA, U.S.A.
}

(Received January 5, 1993)

\begin{abstract}
We have studied the heterogeneous Wurtz-type polymerization of a number of substituted dichlorosilanes at low temperatures as a function of solvent, metal reductant, reaction temperature, reagent stoichiometry, and additives. At $65^{\circ} \mathrm{C}$, aryl dichlorosilanes rapidly produce polymer while dialkyl substituted monomers do not in the absence of additives. While the rate of polymerization of the former is relatively insensitive to steric effects, it is greatly reduced by the presence of electron donating substituents. Reaction temperature is an important variable with higher temperatures producing lower yields of multimodal products. At $65^{\circ} \mathrm{C}$ substituted dichlorosilanes produce high molecular weight products at low monomer conversions independent of reagent stoichiometry in a manner more consistent with a chain growth process. Polymerization in the presence of potential hydrogen donating solvents and additives produced no evidence for hydrogen atom chain transfer which would be expected if trappable silyl radicals are intermediates. The evidence is more consistent with silyl anions rather than silyl radicals as the propagating species in the heterogeneous polymerization.
\end{abstract}

KEY WORDS Polysilanes / Low Temperature Wurtz Coupling / Synthesis

/ Mechanistic Studies /

Substituted silane polymers, i.e., materials which contain only tetravalent silicon atoms in the polymer backbone, represent an interesting class of radiation-sensitive polymers with unusual electronic properties. ${ }^{1-3}$

The earliest synthetic preparation of substituted silane polymers involved the modified Wurtz coupling of dichlorodiphenylsilane. ${ }^{4}$ More recently, transition metal initiated dehydrogenative coupling of silanes has provided a route to low molecular weight silane oligomers. ${ }^{5,6}$ Sakurai et $a l .{ }^{7}$ have reported the anionically initiated polymerization of a variety of masked disilene derivatives as a route for providing molecular weight and dispersity control while retaining terminal anionic reactivity for functionalization. Another anionic route involving the ring-opening polymerization of strained ring cyclosilanes has also been recently reported. ${ }^{8}$
In spite of synthetic advances, the heterogeneous Wurtz-type coupling remains the most general and perhaps least understood procedure for the preparation of high molecular weight linear polysilane derivatives. When run in "inert" solvents boiling above the melting point of sodium metal $\left(97.5^{\circ} \mathrm{C}\right)$, the polymer distribution is often complex and polymodal. ${ }^{1-3,9,10}$ The yield of substituted silane polymer is often low and cyclic oligosilanes are major byproducts. Under these conditions, the polymer yield and molecular weight distribution are acutely sensitive to substituents, order of reagent addition, solvent additives, etc. ${ }^{1}$ Early studies by Zeigler et al. ${ }^{11}$ on the polymerization of dichloromethylphenylsilane (DCMPS) using inverse addition (i.e., sodium dispersion added to monomer) suggested that the heterogeneous nature of the media was responsible for the dispersion in the polymer 
molecular weights. Improvements in the high temperature polymerization process have been achieved by the addition of dipolar additives $^{1,12,13-15}$ such as polyethers, cryptands and crown ethers, albeit often at the expense of polymer molecular weight. These techniques, while they often improve the reproducibility, yields, polymer dispersities, etc., are generally restricted to the preparation of dialkyl polymers.

Recently a number of low temperature polymerization techniques applicable to the polymerization of aryl and, in some instances, alkyl monomers have been reported, ${ }^{16-19}$ all utilizing the "normal" addition mode (i.e., addition of monomer to the metal dispersion). These techniques often employ reaction temperatures below the melting point of sodium $^{16-18}$ or use liquid sodium-potassium alloys. ${ }^{19}$

The first description of a low temperature Wurtz-type polymerization procedure for the preparation of aryl polysilanes was by Matyjaszewski and co-workers, who showed that high molecular weight poly(methylphenylsilane( (PMPS) could be prepared at room temperature using strong ultrasonic activation. ${ }^{16}$ Although this technique may yield essentially monomodal PMPS, there are also certain drawbacks. High intensity ultrasonic sources are necessary. The polymer dispersities depend on ultrasound power ${ }^{20}$ and high molecular weight polymers are rapidly degraded by the ultrasonic radiation. We have reported that very high molecular weight aryl polysilanes could be prepared using dispersed sodium in inert solvents at reaction temperatures between $50-65^{\circ} \mathrm{C}^{17}$ With vigorous stirring, ultrasonic activation is not necessary, and the polymerizations go to completion, even though the sodium is not liquid under the reaction conditions. Recently, Zeigler et al. ${ }^{19}$ have reported similar resulrs using liquid $\mathrm{NaK}$ alloy. The preparation of some polysilanes in good yield using sodium sand in diethyl ether employing a crown ether additive has also been reported by Jones et al. ${ }^{18}$ The utility of the low temperature procedures, particularly for large scale runs, stimulated us to study the process in more detail.

\section{RESULTS}

Although most dichlorosilanes have been polymerized above the melting point of sodium metal, ${ }^{1,2}$ we have recently shown the lower polymerization temperatures often offer better process control and superior products. ${ }^{17} \mathrm{At}$ $65^{\circ} \mathrm{C}$ in toluene solution, the rate of disappearance of most aryl dichlorosilane monomers is very rapid (Figure 1). The characteristic blue-purple color appears during the addition of monomer to the sodium dispersion. Within 10 minutes after the monomer addition, the polymerization has advanced and the amount of monomer has decreased to $<20 \%$ of the original. The bulk of the nonpolymeric silicon containing products is composed of oligomers (cyclic and linear). Four, five and six membered rings are present in the cyclic oligomers. Whereas aryl substituted dichlorosilanes react rapidly with sodium at $65^{\circ} \mathrm{C}$, the dialkyl dichlorosilanes react only slowly and inefficiently in the absence of additives. The

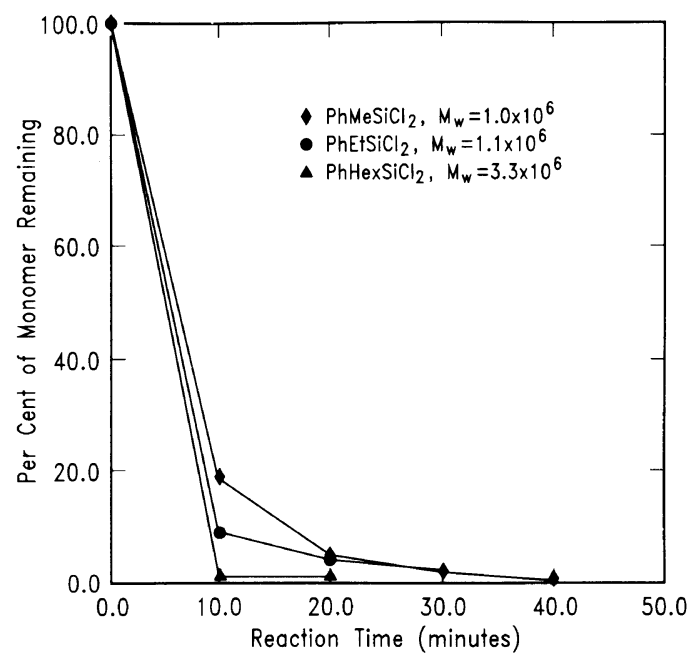

Figure 1. Rate of disappearance of arylalkyldicholorosilane monomers at $65^{\circ} \mathrm{C}$. 
addition of dipolar solvents such as diglyme or catalysts such as crown ethers (15-crown-5, etc.) accelerates the rate of dialkyl monomer disappearance and leads to the formation of high polymer. The effect of additive incorporation on the rate of disappearance of dichlorodi- $n$-hexylsilane (DCDHS) is shown in Figure 2. Even in the presence of additives, the time necessary for the complete consumption of the dialkyl monomers is measured in hours, while that for simple dichloroarylalkyl silanes is dramatically less. In the aryl series, the rate of disappearance of monomer also depends somewhat on structure (i.e., electron-rich monomers are intrinsically less reactive). Figure 3 shows that the alkoxyphenyl substituted monomers are much less reactive than simple phenyl derivatives. In collecting the data in Figures $1-3$, no aliquots were removed before the monomer addition was complete. For this reason, $t=0$ in the figures represents the point where the addition was complete. The molecular weights reported are those of precipitated polymer after complete consumption of monomer.

Although dialkyl substituted monomers do not seem to polymerize efficiently at $65^{\circ} \mathrm{C}$ using sodium metal alone, they do copolymerize with aryl substituted monomers. For example, even though dichloromethylpropylsilane (DCMPS) is relatively stable under the low temperature polymerization conditions (e.g., $>80 \%$ of the monomer remains after $20 \mathrm{~h} 65^{\circ} \mathrm{C}$ ), the subsequent addition of dichloroethylphenylsilane (DCEPS) causes rapid copolymerization with the isolation of a high molecular weight copolymer. At a feed ratio of $1.5 / 1$ (DCEPS/ DCMPS), the copolymer isolated $(12 \%)$ contained the respective monomer units in a ratio of $\sim 7: 1\left(M_{w} 374000, M_{w} / M_{n}=3.1\right)$. Increasing the quantity of the dialkyl monomer in the feed ratio to $1 / 2$ results in increased incorporation of the less reactive dialkyl monomer in the copolymer (monomer ratio in copolymer $\sim 2.5: 1$ ).

The polymer molecular weights produced in

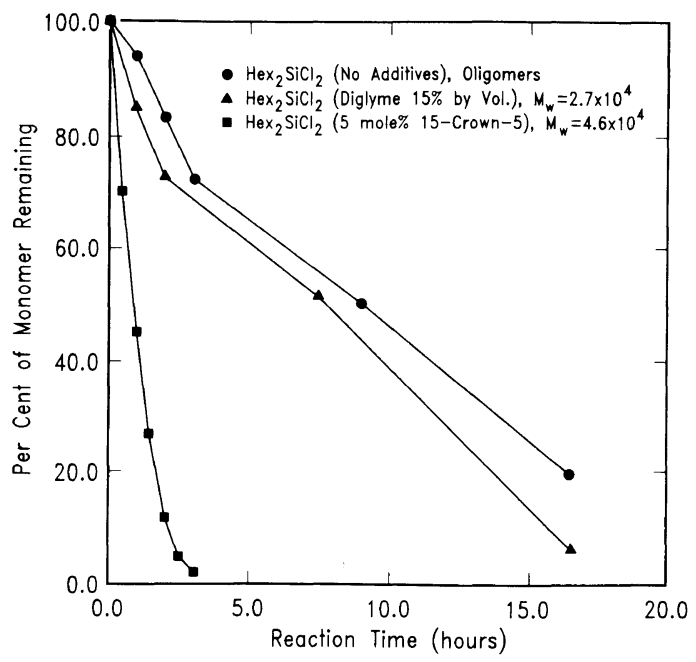

Figure 2. The effect of additives in the polymerization of dichloro-di- $n$-hexylsilane at $65^{\circ} \mathrm{C}$.

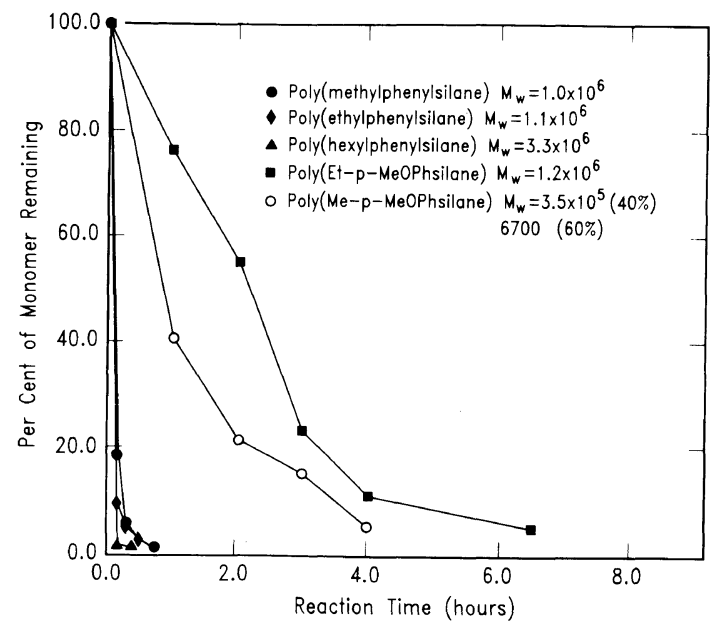

Figure 3. Substituent effects on the rate of disappearance of arylalkyldichlorosilane monomer in the Wurtz-type polymerization at $65^{\circ} \mathrm{C}$.

the polymerization of dichloro-di- $n$-hexylsilane (DCDHS) in the presence of $5 \mathrm{~mol} \% 15$-crown5 and DCEPS alone at $65^{\circ} \mathrm{C}$ were monitored by GPC as a function of monomer conversion (determined by GLPC analysis) and the results are shown in Figure 4.

The controlled nature of the polymerization of dichloroarylalkyl silanes at low temperatures allows this process to be studied in more detail. 
Table I shows the results for the polymerization of a number of monomers under a variety of reaction conditions. All of the data in this table pertain to the normal addition mode of monomer to the preformed sodium dispersion. In most cases, the monomer addition rate was

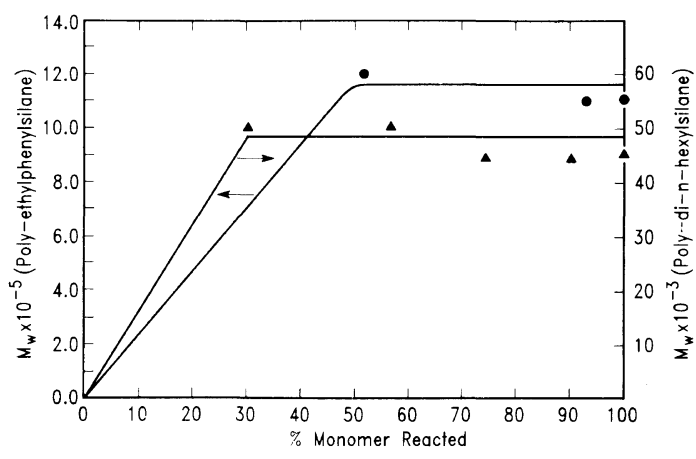

Figure 4. Isolated high polymer molecular weights as a function of monomer conversion at $65^{\circ} \mathrm{C}$. maintained at $0.5 \mathrm{mmol} / \mathrm{min}$ except for entry 3 where the addition rate was increased twofold. For those experiments employing benzene as the solvent, the sodium was first dispersed in toluene at $110^{\circ} \mathrm{C}$, and the solvent subsequently replaced by cannulation.

The variables in this part of the study were solvent, metal dispersion, reaction temperature, rate of addition and reactant stoichiometry. The polymers were purified by reprecipitation from toluene solution by the addition of methanol. For those reactions employing $\mathrm{NaK}$, the alloy contained $\sim 15 \mathrm{wt} \%$ of $\mathrm{K}$. An alloy of this composition is reported to melt around $40^{\circ} \mathrm{C}^{21}$ and is therefore liquid at the polymerization temperature $\left(65^{\circ} \mathrm{C}\right)$. To minimize the effects of scatter in the data, most reactions were repeated more than once and the yields, molecular weights and polymer dispersities were averaged. For example, the

Table I. Wurtz-type polymerization of arylalkyldichlorosilanes

$$
\mathrm{ArRSiCl}_{2}+\mathrm{Metal} \stackrel{\Delta}{\longrightarrow}(\mathrm{ArRSi})_{n}+\mathrm{MetCl}
$$

\begin{tabular}{|c|c|c|c|c|c|c|c|c|}
\hline Entry $^{a}$ & $\mathrm{Ar}$ & $\mathbf{R}$ & Solvent & Metal $^{\mathrm{b}}$ & $\frac{\text { Temp }}{{ }^{\circ} \mathrm{C}}$ & $\begin{array}{c}\text { Yield } \\
\%\end{array}$ & $M_{w} \times 10^{-3 c}$ & $M_{w} / M_{n}$ \\
\hline 1 & $\mathrm{Ph}$ & $\mathrm{Me}$ & Toluene & $\mathrm{Na}$ & 65 & 9 & 1367 & 2.4 \\
\hline 2 & $\mathrm{Ph}$ & Et & Toluene & $\mathrm{Na}$ & 65 & 14 & 975 & 2.4 \\
\hline $3^{\mathrm{d}}$ & $\mathrm{Ph}$ & Et & Toluene & $\mathrm{Na}$ & 65 & 16 & 1250 & 3.0 \\
\hline 4 & $\mathrm{Ph}$ & Et & Toluene & $\mathrm{Na}^{\mathrm{e}}$ & 65 & 14 & 1800 & 4.0 \\
\hline 5 & $\mathrm{Ph}$ & $n$-Hexyl & Toluene & $\mathrm{Na}$ & 65 & 21 & 2752 & 2.2 \\
\hline 6 & $\mathrm{Ph}$ & $\mathrm{Et}$ & Toluene & $\mathrm{NaK}^{\mathrm{f}}$ & 65 & 8 & 821 & 2.6 \\
\hline 7 & $\mathrm{Ph}$ & Et & Benzene & $\mathrm{NaK}^{\mathrm{f}}$ & 65 & 7 & 727 & 3.7 \\
\hline 8 & $\mathrm{Ph}$ & Et & Toluene & $\mathrm{Na}$ & 90 & 4 & 679 & 3.1 \\
\hline 9 & $\mathrm{Ph}$ & Et & Toluene & $\mathrm{Na}$ & 110 & 8 & $\begin{array}{r}1100(12) \\
56(45) \\
12(43)\end{array}$ & $\begin{array}{l}1.5 \\
1.3 \\
1.3\end{array}$ \\
\hline 10 & $\mathrm{Ph}$ & Et & $n$-Butylbenzene & $\mathrm{Na}$ & 65 & 16 & 708 & 2.6 \\
\hline 11 & $\mathrm{Ph}$ & Et & Anisole & $\mathrm{Na}$ & 65 & 5 & $\begin{array}{r}1500(26) \\
130(71)\end{array}$ & $\begin{array}{l}1.4 \\
1.6\end{array}$ \\
\hline 12 & $\mathrm{Ph}$ & Et & Benzene & $\mathrm{Na}$ & 65 & 15 & 917 & 2.2 \\
\hline
\end{tabular}

a Monomer addition rate $0.5 \mathrm{mmol} / \mathrm{min}$ unless otherwise noted; monomer concentration $0.4 \mathrm{M}$.

b Ratio $\mathrm{Na} /$ monomer $=2.2$ unless otherwise noted.

c Molecular weights were measured by GPC and are relative to polystyrene standards.

${ }^{d}$ Monomer addition rate $1.0 \mathrm{mmol} / \mathrm{min}$.

e Molar ratio of $\mathrm{Na} /$ monomer $\sim 5$.

f Weight \% potassium in the alloy was $15 \%$. 
Table II. Wurtz-type polymerization in the presence of various additives

$$
\mathrm{ArRSiCl}_{2}+\mathrm{Metal} \stackrel{\Delta}{\longrightarrow}+\mathrm{ArRSi}_{-}+\mathrm{MetCl}
$$

\begin{tabular}{|c|c|c|c|c|c|c|c|c|}
\hline Entry $^{\mathbf{a}}$ & $\mathrm{Ar}$ & $\mathrm{R}$ & Metal & $\frac{\text { Temp }}{{ }^{\circ} \mathrm{C}}$ & Additive & $\frac{\text { Yield }}{\%}$ & $M_{w} \times 10^{-3 \mathrm{c}}$ & $M_{w} / M_{n}$ \\
\hline 1 & $\mathrm{Ph}$ & $\mathrm{Me}$ & $\mathrm{Na}$ & 65 & - & 7 & 670 & 2.3 \\
\hline 2 & $\mathrm{Ph}$ & $\mathrm{Et}$ & $\mathrm{Na}$ & 65 & - & 14 & 975 & 2.7 \\
\hline 3 & $\mathrm{Ph}$ & $\mathrm{Et}$ & $\mathrm{Na}$ & 65 & TES $^{\mathrm{c}}$ & 12 & 861 & 2.8 \\
\hline 4 & $\mathrm{Ph}$ & $\mathrm{Et}$ & $\mathrm{Na}$ & 65 & $\mathrm{TPS}^{\mathrm{d}}$ & 16 & 1150 & 3.1 \\
\hline 5 & $\mathrm{Ph}$ & $\mathrm{Me}$ & $\mathrm{Na}$ & 65 & TPS & 6 & 858 & 3.3 \\
\hline 6 & $\mathrm{Ph}$ & $\mathrm{Et}$ & $\mathrm{Na}$ & 65 & Anisole $^{e}$ & 12 & 1070 & 2.6 \\
\hline 7 & $\mathrm{Ph}$ & Et & $\mathrm{Na}$ & 65 & TTMSS $^{f}$ & 16 & 742 & 2.2 \\
\hline 8 & $\mathrm{Ph}$ & $\mathrm{Et}$ & $\mathrm{Na}$ & 65 & 1,4-CHD ${ }^{\mathrm{g}}$ & 14 & 765 & 3.0 \\
\hline 9 & $\mathrm{Ph}$ & $\mathrm{Et}$ & $\mathrm{NaK}^{\mathbf{h}}$ & 65 & 1,4-CHD & 9 & 1900 & 2.6 \\
\hline 10 & $\mathrm{Ph}$ & $\mathrm{Et}$ & $\mathrm{Na}$ & 65 & $\mathrm{DMS}^{\mathrm{i}}$ & - & - & - \\
\hline 11 & $\mathrm{Ph}$ & Et & $\mathrm{Na}$ & 65 & PTMSE $^{\mathbf{j}}$ & 14 & 410 & 2.5 \\
\hline
\end{tabular}

a Solvent-toluene, monomer concentration after addition $0.4 \mathrm{M}$. monomer addition rate $0.5 \mathrm{mmol} / \mathrm{min}$, ratio $\mathrm{Na}$ /monomer (2.2).

b Molecular weights were determined by GPC analysis and are based on polystyrene standards.

c Triethylsilane, molar ratio of additive/monomer (2.5).

d Triphenylsilane (2.2).

e Anisole (0.25).

f Tris-trimethylsilylsilane (1.8).

g 1,4-Cyclohexadiene (2.5).

h $15 \mathrm{wt} \%$ potassium.

i Dimethyl sulfate $(0.25)$.

j Phenyltrimethyl silylether (0.25).

data listed in Table I for the homopolymerization of $\mathrm{PhEtSiCl}_{2}$ at $65^{\circ} \mathrm{C}$ (entry 2) are an average of more than ten individual runs. The polymerization temperatures were monitored internally using a thermocouple and the variance was $\pm 10^{\circ} \mathrm{C}$. Maintenance of the internal temperature beyond these limits was difficult because of the exothermicity of the reaction and was particularly troublesome for large scale runs involving aryl substituted monomers.

We have also studied the effect of potential chain transfer or chain terminating additives on the polymerization of DCEPS at $65^{\circ} \mathrm{C}$ and the results are shown in Table II. Again, as before, the data in Table II generally represent averages based on multiple runs. The experiments with the additives were performed in the following manner. The sodium was dispersed in the solvent if the boiling point was above the melting point of $\mathrm{Na}$. When lower boiling solvents (i.e., benzene) were required, toluene was used to disperse the sodium and was decanted from the dispersion and replaced by benzene. The additive was added to the dispersion, the mixture heated to the desired temperature and a $\sim 50 \mathrm{vol} \%$ solution of the monomer in the reaction solvent was added with a syringe pump at a rate of $0.5 \mathrm{mmol}$ of monomer per minute. The rate of disappearance of monomer was not significantly changed by the presence of the additives. Although we have concentrated mainly on the sodium-initiated polymerization, one experiment using $\mathrm{NaK}$ alloy is also included. The molecular weights and polymer dispersities were determined on samples after precipitation and drying. All polymerization reactions were continued for at 
least 30 minutes after all of the monomer had been completely consumed (GLPC analysis).

\section{EXPERIMENTAL}

Aromatic solvents were distilled from sodium ribbon immediately before use. The dichlorosilane monomers were purchased from Huls America Petrarch Systems and distilled from calcium hydride under vacuum. The diglyme (Aldrich Chemical Co., "sure seal") was used without purification. 15-crown-5 (Aldrich Chemical Co.) was distilled from $\mathrm{CaH}_{2}$ before use. Tris-trimethylsilylsilane was purchased from Fluka and was also distilled from $\mathrm{CaH}_{2}$. Gas-liquid chromatographic analyses (GLPC) were performed on a Hewlett Packard model 5736A instrument using $6^{\prime} \times 1 / 4^{\prime \prime}$ glass columns packed with $10 \%$ SE-30 on Chromasorb W. Polymer molecular weight determinations were conducted on a Waters Instruments Model 150C GPC with a refractive index detector and are referenced to polystyrene standards.

General Polymerization Procedure: Poly(ethylphenylsilane) (PEPS)

Into a $125 \mathrm{ml}$ resin flask sealed with an O-ring joint, equipped with a high speed mechanical stirrer driving a multiblade Teflon stirring paddle, Argon inlet and reflux condenser was placed $0.49 \mathrm{~g}(21.4 \mathrm{mmol})$ of clean $1 / 8^{\prime \prime}$ diameter sodium spheres. The sodium was dispersed in $15 \mathrm{ml}$ of dry toluene at reflux (stirrer speed $750-850 \mathrm{rpm}$ ) until a fine sand was produced. After cooling, the sodium on the sides of the flask was washed down with $5 \mathrm{ml}$ of toluene and the flask immersed in a bath at $65^{\circ} \mathrm{C}$. The internal temperature was monitored with a thermocouple and a solution of $2.0 \mathrm{~g}(9.75 \mathrm{mmol})$ dichloroethylphenyl silane freshly distilled from calcium hydride in $2 \mathrm{ml}$ of toluene was added dropwise via a syringe pump over 25 minutes. The reaction mixture was stirred vigorously for $1 \mathrm{~h}$ at $65^{\circ} \mathrm{C}$ after the addition.
At this point, the monomer was completely consumed as determined by GLPC analysis. After cooling to room temperature, the reaction mixture was decomposed by the successive addition of $10 \mathrm{ml}$ of toluene, $2 \mathrm{ml}$ of isopropyl alcohol, and $45 \mathrm{ml}$ of additional toluene. The salts were filtered and rinsed with toluene. The combined toluene fractions were washed twice with water and dried over $\mathrm{MgSO}_{4}$. After removing the drying agent, the polymer was precipitated with $30 \mathrm{ml}$ of methanol and the solid filtered. The polymer was dried $\left(60^{\circ} \mathrm{C} / 100\right.$ Torr $)$ to yield $0.16 \mathrm{~g}(12 \%), M_{w} 1480000, M_{w} / M_{n}=2.4$.

Poly(methylphenylsilane) (PMPS). 9\%; ${ }^{1} \mathrm{H}$ NMR (THF- $\left.d_{8}\right) 6.3-7.2(\mathrm{~m}, 5 \mathrm{H}),(0.5-(-0.8)$, $3 \mathrm{H}) ;{ }^{13} \mathrm{C}$ NMR (tetrahydrofuran $\left(\mathrm{THF}-d_{8}\right)$ $128.0,127.8,135.7,137.2$, and - 5.8; IR (neat) $3067,3047,2956,2895,2362,2337,1427,1408$, $1245,1097,781,752,730,695$, and $668 \mathrm{~cm}^{-1}$; UV (THF) $341 \mathrm{~nm}\left(\varepsilon_{\mathrm{SiSi}}=8300\right)$.

Poly(ethylphenylsilane) (PEPS). $12 \% ;{ }^{1} \mathrm{H}$ NMR $\left(\mathrm{C}_{6} \mathrm{D}_{6}\right) \delta 6.5-7.35$ (brm, 5H, aromatics), $0.5-1.3$ (br m, 5H, ethyl); ${ }^{13} \mathrm{C}$ NMR $\left(\mathrm{CDCl}_{3}\right) \delta 137.1,136.1,133.8,127.0,10.0,6.0$; IR (film) 3070 (w), 3046 (w), 2957 (w), 2930 $(\mathrm{w}), 1426(\mathrm{~m}), 732(\mathrm{~m}), 698(\mathrm{~s})$, and $671(\mathrm{w})$ $\mathrm{cm}^{-1}$; UV (THF) $\lambda_{\max } 342 \mathrm{~nm}\left(\varepsilon_{\mathrm{SiSi}}=11,200\right)$ Anal. Calcd for $\mathrm{C}_{8} \mathrm{H}_{10} \mathrm{Si}: \mathrm{C}, 71.57 \% ; \mathrm{H}, 7.51 \%$; Si, $20.92 \%$; Found: C, 71.47\%, H, 7.45\%; Si, $20.91 \%$.

Poly(n-hexylphenylsilane) (PHPS). $21 \% ;{ }^{1} \mathrm{H}$ $\operatorname{NMR}\left(\mathrm{C}_{6} \mathrm{D}_{6}\right) \delta 6.6-6.7$ (br m, 5H; aromatics), 1.2-1.7 (brm, 13H; hexyl); ${ }^{13} \mathrm{C}$ NMR $\left(\mathrm{CDCl}_{3}\right) \delta 135.9$ (br), 127.1 (br), 33.3, 30.9, 26.4, 22.7, 14.1; IR (film) 3080 (w), 2982 (m), $2944(\mathrm{~s}), 2882(\mathrm{~m}), 2866(\mathrm{~m}), 1426(\mathrm{~m}), 10905$ (m), $683(\mathrm{~s})$, and $674(\mathrm{~s}) \mathrm{cm}^{-1}$; UV (THF) $\lambda_{\max } 348 \mathrm{~nm}\left(\varepsilon_{\mathrm{SiSi}}=11,400\right)$. Anal. Calcd for $\mathrm{C}_{12} \mathrm{H}_{19} \mathrm{Si}: \mathrm{C}, 75,32 \% ; \mathrm{H}, 10.01 \% ; \mathrm{Si}, 14.68 \%$. Found: C, $75.25 \%$; H, 9.83\%; $\mathrm{Si}, 14.68 \%$.

\section{Poly(di-n-hexylsilane). ${ }^{1} \mathrm{H} \quad \mathrm{NMR} \quad\left(\mathrm{C}_{6} \mathrm{D}_{6}\right)$}


$\delta 1.57$ (br m, 20H), 1.05 (br m, 6H); ${ }^{13} \mathrm{C}$ NMR $\left(\mathrm{CDCl}_{3}\right) \delta 34.3,31.7,27.4,22.7,14.9$, and 13.9; UV (cyclohexane) $\lambda_{\max } 317 \mathrm{~nm}\left(\varepsilon_{\mathrm{SiSi}}=9500\right)$.

For those runs where additives were employed, they were added to the reaction flask after the dispersion had cooled to room temperature prior to the addition of the monomer.

\section{DISCUSSION}

The polymerization of $\mathrm{PhMeSiCl}_{2}$ by sodium at $65^{\circ} \mathrm{C}$ proceeds without a significant induction period. The rate of disappearance of the monomer is rapid and is complete within 30 minutes after the completion of monomer addition as shown in Figure 1. The molecular weight of the polymer produced in this process is high and the polymer molecular weight distribution monomodal. Other dichloroarylalkyl silane monomers behave similarly under these conditions and rapidly produce high polymers. Surprisingly, the increased steric hindrance caused by replacement of methyl group in $\mathrm{PhMeSiCl}_{2}$ by larger alkyl substituents seems not to adversely effect the polymerization. The rates of disappearance of $\mathrm{PhRSiCl}_{2}(\mathrm{R}=\mathrm{Et}, n$-hexyl) are also shown in Figure 1 in comparison with the data for $\mathrm{PhMeSiCl}_{2}$. In fact, the substitution of longer alkyl chains for methyl often leads to improved yields of very high molecular weight polymer.

Dichlorodialkyl silanes yield very low yields of high polymer at $65^{\circ} \mathrm{C}$ in the absence of a activating additive. For example, dichloro-di$n$-hexylsilane is consumed only slowly at $65^{\circ} \mathrm{C}$, as shown in Figure 2, and the yield of high polymer isolated was minuscule $(<1 \%)$. The addition of diglyme increases the rate of disappearance of monomer somewhat, and results in the production of high polymer. The effect of crown ethers was much more dramatic with complete monomer consumption occurring within a few hours for 15-crown-5 and led to the production of high molecular weight polymer. Although dichlorodialkyl silanes do not effectively homopolymerize at $65^{\circ} \mathrm{C}$ in the absence of additives, copolymers are readily produced with aryldichlorosilanes. For example, the copolymerization of DCEPS and dichloromethyl- $n$-propyl silane (molar ratio $1.5 / 1)$ produced a monomodal copolymer in $12 \%$ yield. Estimates of the copolymer composition $\left({ }^{1} \mathrm{H}\right.$ NMR, ${ }^{13} \mathrm{C}$ NMR) suggested that the alkyl monomer was incorporated to the extent of approximately $15 \%$. Increasing the amount of dichlorodialkylsilane in the monomer feedstock resulted in an increased incorporation into the isolated copolymer. For example, the copolymer isolated from the polymerization of $\mathrm{PhEtSiCl}{ }_{2}-\mathrm{MePrSiCl}_{2}$ (molar ratio $1 / 2$ ) contained $\sim 30 \%$ of the dialkyl monomer unit.

The rate of disappearance of aryl dichlorosilane monomers under the reaction conditions depends on substitution as shown in Figure 3. Electron donor substituents such as alkoxy slow the polymerization considerably and complete consumption of the monomer requires several hours. In spite of the substantially lower rates of disappearance for the methoxy substituted monomers, high polymers are produced in each case, although a bimodal distribution was observed for the polymer isolated from the polymerization of dichloro-( $p$-methoxyphenylmethyl)silane (see Figure 3). Since the alkoxy substituted monomers are more electron-rich than their unsubstituted counterparts, one might anticipate that surface-mediated electron transfer from sodium would be slower for these materials. Nucleophilic substitution of phenylhalosilanes is also slowed dramatically by the presence of electron donating substituents. ${ }^{22,23}$ For simple nucleophilic substitution on substituted silanes, $\rho^{*}$ values ranging from $+0.52-4.0$ have been measured and depend both on the nucleophile and the leaving group.

It has been suggested that the polymerization of dichlorosilane derivatives using molten sodium to produce high molecular weight 
linear polysilanes more closely resembles a chain growth rather than a step growth process. ${ }^{10}$ This conclusion was based mainly on the production of high polymer at low monomer conversions and the insensitivity of the process to reagent stoichiometry. Similar conclusions apply to the polymerization process at $65^{\circ} \mathrm{C}$, far below the melting point of sodium. Figure 4 shows a plot of the molecular weights of poly(ethylphenylsilane) (PEPS) and poly(di- $n$-hexylsilane) (PDHS) as a function of monomer conversion. In each case, high molecular weight polymer is produced at low monomer conversions and the polymer molecular weights and polymer dispersitivities are essentially unchanged with increased reaction times. In the case of $\mathrm{PhEtSiCl}_{2}$, analysis earlier than $\sim 50 \%$ monomer conversion is difficult due to the rapid rate of monomer consumption. Samples removed immediately after the monomer addition was complete already showed substantial monomer conversion. However, when samples were removed and quenched during the addition, analysis showed that high polymer was also produced very early in the polymerization process! Similarly, the isolated polymer molecular weights were very high even when the molar ratio of sodium/monomer was as high as $5 / 1$ (see entry 4 , Table I).

For the monomer selected for study (i.e., the polymerization of DCEPS) neither the reagent stoichiometry or the rate of monomer addition had much effect on the yield, molecular weight or the dispersivity of the polymer (entries 3 and 4). Temperature, however, appears to be a critical variable. At room temperature, the disappearance of the monomer is very slow and the reaction is of limited preparative value. Under these conditions, the heterogeneous mixture also fails to assume the blue-purple color characteristic of the higher temperature runs which is now associated with the generation of colloidal sodium ${ }^{24}$ and sodium chloride. The color, however, appears rapidly when the unreacted mixture is subsequently heated, and high polymer is ultimately isolated. Temperatures between $50-65^{\circ} \mathrm{C}$ seem ideal for the polymerization process and result in the rapid consumption of monomer, the formation of a characteristic blue color and the isolation of high molecular weight, monomodal polymer. Higher reaction temperatures (albeit still below the melting point of sodium), seem to cause substantially lower yields of slightly lower molecular weight material (entry 8 ). The reaction in toluene at reflux $\left(110^{\circ} \mathrm{C}\right)$ produces a characteristic multimodal polymer distribution (entry 9) that is similar to that obtained in the polymerization of $\mathrm{PhMeSiCl}_{2}$ where the very high molecular weight component is a relatively minor component.

At $65^{\circ} \mathrm{C}$, the use of the liquid $\mathrm{NaK}$ alloy $(15 \mathrm{wt} \% \mathrm{~K})$ seems to offer no significant advantages. The results shown for both toluene and benzene solvents (entries 6 and 7) are comparable. This is surprising in light of the report of Zeigler et al. ${ }^{19}$ who find that for $\mathrm{NaK}$ of similar composition, the use of toluene solvent greatly reduces the polymer molecular weight in the polymerization of $\mathrm{PhMeSiCl}_{2}$ relative to that observed in benzene. Perhaps the fact that these authors apparently conducted their polymerizations at solvent reflux is responsible for this difference. More difficult to rationalize is the observation by these authors that sodium dispersion in benzene produces PMPS polymer of much lower molecular weight $\left(M_{w} 34000\right)$. In our hands, at $65^{\circ} \mathrm{C}$ in toluene, high molecular weight PMPS polymer $\left(M_{w} 1.3 \times 10^{6}\right)$ was isolated in $9 \%$ yield. Again, perhaps it is the different reaction temperatures $\left(65 \mathrm{vs} .80^{\circ} \mathrm{C}\right)$ which leads to the difference. If so, the polymerization temperature is truly a very important variable in the heterogenous Wurtz-type polymerization process. Another possible source of this discrepancy is the nature of the sodium dispersion itself, since Zeigler et al. ${ }^{19}$ apparently used commercial dispersion while ours was freshly prepared. We have also surveyed a number of aromatic solvents in the polymerization of 
DCEPS at $65^{\circ} \mathrm{C}$. In this regard, we observe little difference in the polymer yields, molecular weights or distributions as the solvent is varied between toluene, $t$-butylbenzene, and benzene (entries 2, 10, and 12). The use of anisole does, however, result in a somewhat lower polymer yield and a strongly bimodal polymer molecular weight distribution (entry 11). Even in this case, however, the isolated polymer fractions are both relatively high molecular weight. It seems likely that the effect of anisole in this case represents a true bulk solvent effect rather than signifying a change in the polymerization mechanism or the onset of significant chain transfer from the solvent (vide infra).

The complexity of the Wurtz-type coupling process has hindered definitive mechanistic studies. At first, it was conjectured that the generation of polymers with distinctly polymodal molecular weight distributions argued for completing mechanisms. ${ }^{10}$ Now it is generally conceded that the polymodal molecular weight distribution may be a result of the heterogeneous nature of the polymerization which proceeds at or near the active sodium surface. ${ }^{11,25}$ Even the heterogeneity itself is complex, since during the polymerization, in addition to the homogeneous liquid there are at least two solid phases (i) high surface, reactive sodium and (ii) insoluble sodium chloride produced during polymerization. If soluble, the polymer may be partitioned between the solution and solid phases in a dynamic equilibrium or alternatively in very poor solvents for the polymer may precipitate forming either a third solid phase or coating the sodium-sodium chloride solid phase(s). The dynamics of absorption-desorption as well as the thermodynamic solubility of the polymer will depend on the substituents, solvent, temperature, molecular weight, etc.

Reactive intermediates variously proposed for the polymerization process include silylenes, disilenes, silyl radicals, sily anions, and even radical anions. It is now generally agreed that free silylenes and disilenes are probably not involved in the chain growth process due to the ineffectiveness of known traps to alter the course of the polymerization. The results of this study support also this conclusion. The effect of dipolar additives, in particular polyethers, crown ethers and cryptands, on the rate of disappearance of monomer and on the yield, molecular weight and polydispersity of the polymer seems most consistent with silyl anionic propagation.

Still the question of the role of silyl radicals in the chain growth and chain extension processes has persisted. This mechanism was first suggested by Zeigler ${ }^{11}$ as an explanation for the low polymer molecular weights obtained from monomers such as dichloromethyl ( $p$-dimethylaminophenyl)silane and dichloromethyl( $p$-methoxyphenyl)silane in toluene. Rapid chain transfer or chain termination of the silyl radicals by hydrogen abstraction leading to low polymer molecular weights was proposed. Consideration of this proposal in terms of the relevant bond energetics is informative. Modern measurements of silicon hydride bond energies suggest that the $\mathrm{SiH}$ bond strength is not much different from the $\mathrm{CH}$ bond energy in alkanes. ${ }^{26}$ Furthermore, the former appears much less responsive to variation with substitution than the latter. In this respect the homolytic bond energies of silane, trimethylsilane and phenylsilane are $90.3,90.3$, and $88.2 \mathrm{kcal} \mathrm{mol}^{-1}$ respectively. ${ }^{26}$ Likewise, halogen substitution has little effect, since the bond energy of trichlorosilane is higher still at $91.3 \mathrm{kcal} \mathrm{mol}^{-1}$. What does significantly effect the $\mathrm{SiH}$ bond energy, however, is catenation with other silicon substituents. This is demonstrated by the fact that the $\mathrm{SiH}$ bond energy of disilane is already $\sim 4 \mathrm{kcal} \mathrm{mol}^{-1}$ lower than silane itself. Unfortunately, $\mathrm{SiH}$ bond energy data for higher silcon catenates are not available, but it seems likely that the bond energy will decrease in rapidly diminishing increments and saturate at a relatively low level of catenation. 
If one reasonably estimates the $\mathrm{SiH}$ bond energy of a polymeric silicon catenate to be around $78-82 \mathrm{kcal} \mathrm{mol}^{-1}$ (even a catenate with pendant phenyl substituents), this suggests that silyl radical hydrogen abstraction from alkanes, alkyl aromatics and simple silanes should be reasonably endothermic. The same argument should apply to ethers for which the measured bond energies are also quite high (e.g., the $\alpha$-carbon-hydrogen bond energy for tetrahydrofuran is $\left.92 \mathrm{kcal} \mathrm{mol}^{-1}\right){ }^{27}$ This would seem to suggest, purely on bond energy arguments, that ethereal solvents or substituents would not be expected to be particularly effective hydrogen atom transfer agents for a growing catenated polysilyl radical. Furthermore, it is known that simple crown ethers present in very low concentrations $(0.25-5 \mathrm{~mol} \%$ based on monomer) will drastically reduce the molecular weight of the polymer produced from $\mathrm{PhMeSiCl}_{2}$ from 1 million or more to a few tens of thousands or less. If this result is caused solely by chain transfer or termination by hydrogen abstraction from the low concentrations of crown ether present, these reagents must be extraordinarily effective chain transfer reagents indeed!

As noted earlier, solvent changes (benzene, $p$-t-butylbenzene, toluene) also have relatively little effect on the course of the polymerization of DCEPS. Toluene is, in principle, a hydrogen atom donating solvent, and the low polymer molecular weight of PMPS in toluene using $\mathrm{NaK}$ has been ascribed to hydrogen atom abstraction from solvent, ${ }^{19}$ although the calculated bond energies suggest that benzylic hydrogen abstraction by a polycatenated silyl radical would probably be endothermic. Even so, the measured rate constant $(300 \mathrm{~K})$ for reaction of triethylsilyl radicals with toluene is substantial $\left(1.2 \times 10^{6} 1 \mathrm{~mol}^{-1} \mathrm{~s}^{-1}\right) .{ }^{28}$ However, the reaction of triethylsilyl radicals with toluene is proposed to proceed by addition to the aromatic ring to generate substituted cyclohexadienyl radicals. ${ }^{28}$ Intermediates of this type have been detected by $\operatorname{ESR}^{29,30}$ and the operation of this addition pathway was confirmed by the isolation of various aromatic substitution products. ${ }^{31-33}$ Although hydrogen abstraction from toluene may be competitive, there is no evidence that this is indeed the case. In fact, the report by Matyjaszewski et al. ${ }^{34}$ that $\mathrm{PhMeSiCl}_{2}$ reacts with potassium in toluene to yield a polymer, which is not a true polysilane, and which contains incorporated toluene units suggests that aromatic substitution rather than abstraction may be the preferred mode of reaction of polysilyl radicals with toluene. Since we do not observe either a solvent effect or solvent incorporation in the polymerization of $\mathrm{PhEtSiCl}_{2}$ at $65^{\circ} \mathrm{C}$, even with $\mathrm{NaK}$, we conclude that the reported $^{19}$ reduction in polymer molecular weight of PMPS in toluene using $\mathrm{NaK}$ must either be substrate specific and/or depend strongly on the reaction temperature.

Silane derivatives containing $\mathrm{SiH}$ bonds have been employed as efficient trapping reagents for divalent silicon species (silylenes) ${ }^{35}$ They have also been studied as chain transfer media in the thermal polymerization of styrene $\mathrm{s}^{36}$ where a chain transfer rate constant of $22 \times 10^{5} \mathrm{~mol}^{-1} \mathrm{~s}^{-1}\left(60^{\circ} \mathrm{C}\right)$ was measured for triethylsilane and the value for triphenylsilane was approximately an order of magnitude higher.

Examination of the data in Table II shows that neither triethylsilane (TES) nor triphenylsilane (TPS) has mush effect on the product molecular weights or polymer yields in the polymerization of either $\mathrm{PhMeSiCl}_{2}$ or $\mathrm{PhEtSiCl}_{2}$. While these experiments probably constitute good evidence against the intermediacy of free silylenes in the polymerization, their relevancy regarding the presence or absence of propagating silyl radicals may be questioned on the basis of bond energetics. What is really needed are additives with very low silicon-hydrogen or carbon-hydrogen bond energies $\left(<80 \mathrm{kcal} \mathrm{mol}^{-1}\right)$ which are themselves stable in the polymerization media and which do not markedly effect the bulk 
solvent properties, even when employed in considerable molar excess.

Examples of such materials are tris-trimethylsilylsilane and 1,4-cyclohexadiene. The former has been proposed as a alternative to tributyltin hydride in the free radical dehalogenation of alkyl halides and the siliconhydrogen bond energy has been measured (78 $\left.\mathrm{kcal} \mathrm{mol}^{-1}\right) .^{37,38}$ The latter has very weak doubly allylic carbon-hydrogen bonds which are prone to abstraction by free radical reagents. The allylic carbon-hydrogen bond energy in cyclohexadiene is very low; around $71 \mathrm{kcal} \mathrm{mol}^{-139}$ Each reagent is inert to sodium in hydrocarbon. solvents at $65^{\circ} \mathrm{C}$ and should cause minimum change in the solvent polarity. Neither additive causes a significant decrease in either the molecular weight or in the high polymer yield when added to the polymerization of DCEPS at $65^{\circ} \mathrm{C}$ (entries 6 and 7). When 1,4-cyclohexadiene is used in conjunction with $\mathrm{NaK}$ alloy, the molecular weight of the isolated polymer actually increases over that obtained in the absence of additive (see Table II, entry 8 vs. entry 7). Since this is the result of a single run, it is not known whether the increase in the molecular weight is statistically significant, but it is quite certain that the molecular weight of the polymer produced is not significantly lower than is observed in the absence of additive.

The failure to detect evidence of significant intermolecular chain transfer or silicon chain termination in the polymerization of DCEPS, even when the thermodynamics for hydrogen abstraction should be more favorable, does not mean that silyl radicals cannot be fleeting intermediates at or near the metal surface. Indeed, it would seem unlikely that radicals are not produced initially on or near the metal surface in the reduction of a substituted dichlorosilane. It does, however, suggest that any oligomeric or polymeric radicals probably do not leave the surface to enter the bulk solution where they would be susceptible to trapping, which would be expected to sig- nificantly broaden the distribution and reduce the molecular weight and yield of high polymer. This is in spite of the fact that the concentration of trapping agent in bulk, and also presumably at the sodium surface, is certainly much greater than the monomer concentration, particularly early in the addition.

By increasing the potential exothermicity of electron transfer to monomer, as well as to chlorinated oligomers and polymers using the more reactive $\mathrm{NaK}$, it was hoped that radicals could be generated further from the metal surface to facilitate radical trapping prior to subsequent reaction. A similar argument has been advanced in the heterogeneous formation of Grignard reagents from alkyl halides and magnesium. ${ }^{40,41}$ For example, the use of alkyl iodides versus alkyl chlorides results in the formation of Grignard reagents and leads to increased quantities of traditional radical-type products. It is assumed that the more exothermic electron transfer can take place over larger distances allowing the intially formed radicals more time for rearrangement and escape. This situation also improves the eficiency of intermolecular radical trapping. In this regard, Matyjaszewski et $a .^{34}$ have reported that the reaction of $\mathrm{PhMeSiCl}_{2}$ with potassium metal in toluene produces a polymer which is not actually a polysilane, but rather incorporates a substantial amount of the solvent. Under similar reaction conditions, sodium produces high molecular weight polysilane. This may be the reason why toluene appears to function as a chain transfer medium in the polymerization of $\mathrm{PhMeSiCl}_{2}$ using $\mathrm{NaK}$ at elevated temperatures $\left(110^{\circ} \mathrm{C}\right),{ }^{19}$ although one might also expect to incorporate toluene units into the polymer under these conditions. However, we have observed no evidence of significant chain transfer or the incorporation of toluene or additive in the polymerization of DCEPS using $\mathrm{NaK}$ at $65^{\circ} \mathrm{C}$ either in toluene solvent alone or in toluene containing 1,4cyclohexadiene.

Our study of the effect of external additives 
on the polymerization of $\mathrm{PhEtSiCl}_{2}$ at $65^{\circ} \mathrm{C}$ failed to produce any evidence of significant chain transfer or termination even for materials that might be expected to be good chain transfer additives based on thermodynamic energy considerations. This suggests that silyl radicals, if intermediates in the Wurtz-type coupling, are confined and react on or near the sodium surface and are short-lived. This proposal is also consistent with the failure to observe ESR signals characteristic of silyl radicals or radical anions in a polymerizing mixture of substituted dichlorosilanes and sodium. ${ }^{24}$ In fact, the only experimental evidence for the involvement of silyl radicals in the Wurtz-type polymerization of dichlorosilanes comes from a study of the polymerization of monomers containing terminal alkenyl substituents. ${ }^{42}$ The 1-hexenyl substituted monomers were particularly instructive as intramolecular silyl radical olefin addition is known to be very fast $(1-3$ orders of magnitude faster than the intermolecular variant $)^{42}$ and hence might be expected to compete with the suface mediated polymerization. In the homopolymerization of dichloro1-hexenylphenyl silane, a very small amount of high polymer is produced $(<1 \%)$ and the oligomeric fraction shows that $75 \%$ of the olefinic functionality is consumed. In this particularly favorable case, silyl radical cyclization apparently occurs early in the catenation sequence. The formation of a small amount of high polymer suggests that if the silyl radicals escape cyclization at the oligomer state, the subsequent polymeric radicals are more rapidly converted to other reactive intermediates (perhaps silyl anions) which can propagate, rather than undergo radical cyclization. This hypothesis would also be consistent with the observed decrease in the reduction potentials of chlorosilanes (and presumably also silyl radicals) with increasing catenation. ${ }^{43}$ The authors conclude that initial SET electron transfer to produce a chlorosilane radical ion is followed by dissociation to yield short-lived silyl radicals which rapidly accept another electron to yield silyl anions that propagate by nucleophilic displacement on another monomer molecule or couple with oligomeric or polymeric chlorosilanes present in the media.

The production of very high molecular weight polymers $\left(M_{n}=0.5-1.0 \times 10^{6}\right.$ Daltons; DP $>3500$ ) itself argues against extensive radical-radical coupling for chain extension, since the reaction between two sterically encumbered radicals should partition between dimerization and disproportionation. In the formation of Grignard reagents by the reaction of magnesium with organic halides, disproportionation has been found to be competitive even for radicals confined to or near the metal surface. ${ }^{41}$ Disproportionation should become more competitive as the steric bulk of the radicals and coupling products increases, based on studies of carbon-centered radicals. ${ }^{44}$ Disproportionation between silyl radicals is also known to be a competitive process. For example, pentamethyldisilanyl radicals disproportionate and recombine about evenly. ${ }^{45}$ There are also many other cases of competitive disproportionation of silyl radicals known in the literature ${ }^{46}$ Furthermore, silyl radicals are clearly intermediates in the exhaustive photodecomposition of silane high polymers ${ }^{4-49}$ and on the basis of isotopic labeling studies, silyl radical disproportionation reactions have been proposed as the primary source of $\mathrm{SiH}$ containing oligomers at room temperature. ${ }^{49}$

The case for propagating anions in the Wurtz-type polymerization seems somewhat better developed. First, there are substantial solvent effects observed in the presence of dipolar solvents or cation complexing species such as crown ethers and cryptands. The effect of these additives is usually a decreased induction period for monomer disappearance, faster consumption of monomer, higher polymer yields, monomodal polymer molecular weight distributions and somewhat lower polymer molecular weights. These results are often realized at very low additive 
concentrations $(0.25-5 \%)$, for crown ethers and cryptands. These effects are shown dramatically in Figure 4 in the polymerization of dichloro-di-hexylsilane in toluene at $65^{\circ} \mathrm{C}$. With no additive, the disappearance of monomer occurs very slowly but little $(<1 \%)$ high polymer is isolated. The addition of $15 \%$ by volume of diglyme increases the rate of monomer disappearance somewhat, and high polymer $\left(M_{w}=27000\right)$ is isolated from the reaction mixture $(12 \%)$. The addition of $5 \mathrm{~mol} \%$ of 15 -crown -5 results in a dramatic increase in the rate of monomer disappearance with complete consumption occurring in only a few hours. The polymer $\left(M_{w}=46000\right)$ isolated from the reaction mixture $(22 \%)$ showed a monomodal molecular weight distribution. The only other products were low molecular weight cyclic oligomers. The effect of crown ethers and cryptands is more understandable if anions are involved in the chain growth process since there is ample precedent for their influence on anionic reactions and polymerizations. ${ }^{50-52}$ It is certainly possible that the presence of cation complexing additives facilitates initial electron transfer from the metal to the monomer, since this constitutes a common step in either the radical or the anionic mechanism and is believed to be a slow step in the sequence. If this is the case, however, it is hard to argue that the presence of complexing additives would not similarly accelerate the subsequent reduction of silyl radicals to silyl anions, while radical-radical coupling reactions should be less sensitive to additives. It also appears, since crown ethers are often effective at concentrations as low as $0.25 \mathrm{~mol} \%$, that the lower polymer molecular weights obtained in the presence of these additives is more consistent with accelerated initiation producing more growing chains rather than chain transfer or termination involving the additive.

Second, there is the formation of substantial quantities of cyclosilanes during the polymerization process. Although Worford has demonstrated that the ratio of cyclics/polymer remains roughly constant during the polymerization of dichlorohexylmethylsilane in the presence of 15 -crown- $5,{ }^{14}$ which suggests a competitive ring/chain process, it is not clear that this is the only source of cyclics in all dichlorosilane polymerizations. In particular, anionic backbiting is implicated as a source of cyclics in the metal-initiated decomposition of silane high polymers in polar solvents or in the presence of cryptands. ${ }^{14,53}$ This process is also accompanied by the formation of various colors said to be characteristic of silyl anions. ${ }^{54}$ The role of silyl anions in end-biting cyclization reactions and under certain reaction conditions in backbiting to produce cyclosilanes from polysilane polymers seems based on ample precedent. Such is not the case for silyl radical intermediates which would require either energetically unfavorable chain transfer from an $\omega$-chlorooligosilyl radical or the statistically improbable cyclication of an $\alpha, \omega$-polysilyldiyl biradical in the absence of an intramolecular homolytic backbiting route to cyclics. It should be mentioned that the latter route has recently been proposed as a source of cyclics in the Wurtz-type polymerization ${ }^{19}$ and 5- and 6-membered cyclosilanes are indeed produced in the high temperature $\left(>200^{\circ} \mathrm{C}\right)$ thermal decomposition of PMPS. ${ }^{53}$ On the other hand, cyclosilanes are not detected in the ultrasonic degradation of high molecular weight PMPS $^{53}$ at or near ambient temperature in toluene solution, a process which presumably involves polymer chain scission and silyl radical formation. ${ }^{55}$ This result suggests that backbiting of free polysilyl chain radicals to form 5- and 6-membered cyclosilanes may not be a competitive process at lower reaction temperatures.

Finally, there is the question of unreactivity of dichlorodialkyl silanes with sodium at low temperatures in nonpolar solvents. This was noted by Matyjaszewski and $\mathrm{Kim}^{16}$ for ultrasonic reactions who also observed that even though dichloro-di- $n$-hexylsilane did not 
homopolymerize under the conditions, it could be copolymerized with $\mathrm{PhMeSiCl}_{2}$. We also have reported the unreactivity of dichlorodialkylsilanes at low temperatures both with and without ultrasonic agitation. ${ }^{17,56}$ For example, at $65^{\circ} \mathrm{C}$ in the absence of ultrasonic agitation, dichloromethylpropyl silane disappeared only slowly over a period of many hours. Workup led to the isolation of high polymer in less than $0.5 \%$ yield. On the other hand, we have found that copolymerization of dichlorodialkyl and aryl alkylsilanes occurs readily at $65^{\circ} \mathrm{C}$ even without ultrasonic agitation. In this regard, dichloromethylpropyl silane readily copolymerizes with $\mathrm{PhEtSiCl}_{2}$ to produce high molecular weight material which is enriched in the aryl substituted monomer. The composition of the respective copolymers remained roughly constant for reaction times varying from $1 \mathrm{~h}$ to more than $60 \mathrm{~h}$ and varied with the comonomer feed ratio.

For the purpose of compositional analysis of the copolymers, ${ }^{13} \mathrm{C}$ NMR was particularly useful, since the respective alkyl resonances for the ethyl, $n$-propyl, and methyl groups in the copolymer are widely separated (5.9 and 11.0 vs. $16-21$ and $-3.1 \mathrm{ppm}$, respectively). ${ }^{1} \mathrm{H}$ NMR is less useful for quantitative analysis, since the resonances for the ethyl group in poly(ethylphenylsilane) (PEPS) are broad at room temperature and strongly overlap those of the $n$-propyl group in the copolymer. The unreactivity of dichlorodialkyl silanes alone and the formation of copolymers from dialkyl and arylalkyl dichlorosilanes using ultrasonic agitation led Matyjaszewski et al. ${ }^{34,53}$ to originally propose that silyl radicals could not be the sole propagating species in the copolymerization reaction. Our results at $65^{\circ} \mathrm{C}$ also support this conclusion. The ready incorporation of a potentially unreactive monomer into a copolymer under the reaction conditions seems more consistent with an anionic mechanism where propagation proceeds by anionic nucleophilic displacement (a process which could incorporate the dialkyl monomer in a competitive fashion) than for a purely radical mechanism. For the latter, at low temperatures one would anticipate the rapid formation of a homopolymer of $\mathrm{PhEtSiCl}_{2}$ (polymerization active) even in the presence of the comonomer feedstock.

On the other hand, it has proved relatively difficult to find an anion specific electrophilic reagent sufficiently stable under the reaction conditions which is capable of intercepting and terminating the growing polysilane anionic chain. Worsfold and Gauthier ${ }^{57}$ have reported the formation of block copolysilanes by the sequential addition of different dichlorosilane monomers, suggesting that the polymerization has certain characteristics of a living anionic polymerization. They also found that isolated homopolymer could be reactivated toward copolymerization after isolation by readdition to sodium and another dichlorosilane monomer. Although the authors state that NMR analysis indicated block copolymer formation, no detailed spectroscopic analysis of the copolymers was reported. Indeed, a detailed structural analysis might be complicated by the known tendency of silicon catenates to undergo equilibration and redistribution in the presence of anionic reagents. ${ }^{54}$

Our attempts to terminate anionic chain ends by the inclusion of an alkylating agent such as dimethyl sulfate into the polymerization media were unsuccessful. Although no visible reaction of dimethyl sulfate with sodium occurred upon mixing, subsequent addition of $\mathrm{PhEtSiCl}_{2}$ produced no color change or the formation of any high polymer (Table II, entry 10). Excess sodium remained after $10 \mathrm{~h}$ at $65^{\circ} \mathrm{C}$ as evidenced by the vigorous reaction upon decomposition with isopropyl alcohol. On the other hand, the polymerization of DCEPS in the presence of low concentrations of phenyltrimethylsilyl ether $(0.25 \mathrm{~mol} / \mathrm{mol}$ monomer $)$ led to the complete consumption of the sodium and the formation of high polymer in $10 \%$ yield (a yield not significantly different from the homopolymerization in the absence of 
additive, see Table II). GPC analyses of three identical runs indicated a monomodal polymer molecular weight distribution with an average $M_{w}$ of 410000 ; a value significantly lower than obtained in the runs without the additive. This reduction in molecular weight does not seem to reflect a bulk solvent effect, since subsequent runs in toluene containing the same molar ratio of anisole to monomer were virtually indistinguishable from the runs in toluene alone (Table II, entry 6). However, polymerization of DCEPS in pure anisole produced a product which was strongly bimodal (Table I, entry 11), an effect which we attribute to a bulk solvent effect rather than competitive chain transfer, since the molecular weight of the high molecular fraction is not significantly reduced nor is the dispersity particularly large. It is possible therefore that the reproducibly lower molecular weights of poly(ethylphenylsilane) obtained in the presence of low concentrations of phenyltrimethylsilyl ether may result from trapping of propagating silyl anions competitively with polymerization but more work is necessary to confirm this hypothesis.

In summary, we have studied the polymerization (normal addition) of a variety of substituted dichlorosilanes at low temperatures without activation by ultrasonic agitation. In most cases, reasonable yields of very high molecular weight, monomodal polymers were obtained from aryl substituted dichlorosilane monomers. Electron donating substituents on the aromatic rings greatly decreased the rate of monomer disappearance but still produced high polymers. Cyclic and linear oligomers comprise the bulk of the soluble silicon containing products. Dialkyl dichlorosilanes disappeared only slowly under the reaction conditions without the formation of significant amounts of high polymer. In the presence of certain dipolar additives, these normally unreactive monomers also rapidly formed monomodal high polymers in reasonable yields. In the regard, crown ethers were much more effective than glyme or diglyme even when incorporated at very much lower concentrations. In all cases, high molecular weight polymer was produced at low conversions in a manner more consistent with a chain growth rather than a step growth process. For the arylalkyl monomers, increasing the length of the alkyl substituent had no significant effect on the rate of monomer disappearance and produced monomodal high molecular weight polymers. This is in spite of the fact that increasing the steric bulk around silicon might be expected to facilitate radical disproportionation reactions relative to recombination if chain growth and extension were purely a radical process. In a detailed study of the polymerization of DCEPS at $65^{\circ} \mathrm{C}$, it was found that changes in solvent from $t$-butylbenzene or benzene to toluene produced comparable results with no evidence of significant chain transfer in the latter solvent. This was true even using $\mathrm{NaK}$ in contrast to a recent report in the literature $^{19}$ which utilized higher reaction temperatures. The addition of trisubstituted silanes did not significantly lower the molecular weights or broaden the molecular weight distributions as would be expected if silylenes were intermediates or if hydrogen atom chain transfer were occurring. Bond energy considerations suggest that hydrogen abstraction from these additives by a catenated polysilyl radical would be endothermic. However, neither was any significant effect observed for additives such as tris-trimethylsilylsilane or 1,4-cyclohexadiene where hydrogen transfer to a catenated silyl radical chain would be expected to be more thermodynamically favorable. This is true even using the more reactive $\mathrm{NaK}$ where electron transfer and radical formation might be expected to take place further from the metal surface. We conclude, therefore, that polysilyl radicals, if produced, are present as fleeting intermediates, probably located on or near the metal surface and do not wander into solution where they would be susceptible to external trapping. We propose, as have others, that the heterogeneous 
Wurtz-type polymerization of dichlorosilanes is best described by anionic propagation and the rate of reduction of both the polymeric chlorides and radicals increases, at least to a point, as the silicon chain length increases. This conclusion is also supported by the fact that dialkyl dichlorosilanes do not homopolymerize significantly at $65^{\circ} \mathrm{C}$, but readily copolymerize with aryl alkyl dichlorosilanes which are more readily reduced by the metal. Although attempts to trap the propagating silyl anions with electrophilic reagents stable under the reaction conditions have not been very successful, the reduced polymer molecular weights obtained in the presence of phenyltrimethylsilyl ether may result from competitive trapping of anionic species.

\section{REFERENCES}

1. R. D. Miller and J. Michl, Chem. Rev., 89, 1354 (1989) and references cited therein.

2. P. West, in "The Chemistry of Organic Silicon Compounds," Part II, John Wiley \& Sons, New York, 1989, Chapter 19 and references cited therein.

3. J. M. Zeigler, Mol. Cryst. Liq. Cryst., 190, 265 (1990).

4. F. S. Kipping, J. Chem. Soc., 119, 830 (1921).

5. (a) J. F. Harrod, in "Inorganic and Organometallc Polymers," ACS Symposium Series 360, M. Zelden, K. J. Wynne, H. R. Allcock, Ed., The American Chemical Society, Washington, DC, 1987, Chapter 7 and references cited therein. (b) J. Y. Corey, X.-H. Zhu, T. C. Bedard, and G. D. Lange, Organomet, 10, 924 (1991).

6. P. L. Watson, F. N. Tebbe, U. S. Patent $4,965,386$ (1990).

7. K. Sakamoto, K. Obata, H. Hirata, M. Nakajima, and H. Sakurai, J. Am. Chem. Soc., 111, 7641 (1989).

8. M. Cypryk, Y. Gupta, and K. Matyjaszewski, J. Am. Chem. Soc., 113, 1046 (1991).

9. P. Trefonas, III, P. I. Djurovich, X.-H. Zhang, R. West, R. D. Miller, and D. Hofer, J. Polym. Sci. Polym. Lett. Ed., 21, 819 (1983).

10. D. J. Worsfold, in "Inorganic and Organometallic Polymers," ACS Symposium Series 360, M. Zelden, K. J. Wynne, and H. R. Allcock, Ed., The American Chemical Society, Washington, DC, 1988, Chapter 8.

11. J. M. Zeigler, Polym. Prepr., 27, 109 (1986).

12. R. D. Miller, J. F. Rabolt, R. Sooriyakumaran, W. Fleming, G. N. Fickes, B. L. Farmer, and H. Kuzmany, in "Inorganic and Organometallic Polymers," ACS Symposium Series 360, M. Zelden, K.
J. Wynne, H. R. Allcock, Ed., The American Chemical Society, Washington, DC, 1988, Chapter 4 and references cited therein.

13. R. D. Miller, D. Hofer, D. R. McKean, G. C. Willson, R. West, and P. T. Trefonas, III, in "Materials for Microlithography," ACS Symposium Series 266, L. F. Thompson and C. G. Willson, Ed., The American Chemical Society, Washington, DC, 1984, Chapter 14.

14. S. Gauthier and D. J. Worsfold, Macromolecules, 22, 2213 (1989).

15. M. Fijino and H. Isaka, J. Chem. Soc., Chem. Commun., 466 (1989).

16. H.-K. Kim and K. Matyjaszewski, J. Am. Chem. Soc., 110, 3321 (1988).

17. R. D. Miller, D. Thompson, R. Sooriyakumaran, and G. N. Fickes, J. Polym. Sci.: A, Polym. Chem., 29, 813 (1991).

18. R. H. Cragg, R. G. Jones, A. C. Swain, and S. J. Webb, J. Chem. Soc., Chem. Commun., 1147 (1990).

19. J. M. Zeigler, L. I. McLaughlin, and R. J. Perry, J. Inorg. Organomet. Polym., 1, 531 (1991).

20. G. J. Price, J. Chem. Soc. Chem. Commun., 1209 (1992).

21. G. L. C. M. van Rossen Hoogendijk van Bleiswijk, Z. An. Chem., 74, 152 (1992).

22. L. M. Sommer, "Stereochemistry, Mechanism and Silicon," McGraw-Hill, New York, 1965.

23. G. Schott, Z. Chem., 6, 361 (1966).

24. R. E. Benfield, R. H. Cragg, R. G. Jones, and A. C. Swain, Nature, 353, 340 (1991).

25. R. G. Jones, R. E. Benfield, R. H. Cragg, and A. C. Swain, J. Chem. Soc., Chem. Commun, 112 (1992).

26. R. Walsh, Acc. Chem. Res., 14, 246 (1981).

27. F. R. Cruckshank and S. W. Benson, J. Am. Chem. Soc., 91, 1289 (1969).

28. C. Chatgilialoglu, K. U. Ingold, and J. C. Scaiano, J. Am. Chem. Soc., 105, 3292 (1983).

29. D. Griller, K. Dimroth, T. M. Fyles, and K. U. Ingold, J. Am. Chem. Soc., 97, 5526 (1973).

30. H. Sakurai, I. Nozue, and A. Hosomi, Chem. Lett., 129 (1976).

31. C. Eaborn, R. A. Jackson, and R. Pearce, J. Chem. Soc., Chem. Commun., 920 (1967).

32. M. J. Perkins, in "Free Radicals," Vol. II, and J. K. Kochi, Ed., John Wiley \& Sons: New York, 1973, $231 \mathrm{ff}$.

33. H. Sakurai and A. Hosomi, J. Am. Chem. Soc., 93, 1709 (1971).

34. K. Matyjaszewski, M. Cypryk, H. Frey, J. Hrkach, H. K. Kim, M. Moeller, K. Ruehl, and M. White, J. Macromol. Sci.-Chem., A28 (11 and 12), 1151, (1991).

35. P. P. Gaspar, D. Holten, S. Koneczny, and J. Corey, Y. Acc. Chem. Res., 20, 329 (1987).

36. J. Curtice, H. Gilman and G. S. Hammond, J. Am. Chem. Soc., 79, 4754 (1957). 
37. C. Chatgilialoglu, D. Griller, and M. Lesage, J. Org. Chem., 53, 3642 (1988).

38. J. M. Kanabus-Kaminska, J. A. Hawari, D. Griller, and C. Chatgilialoglu, J. Am. Chem. Soc., 109, 5267 (1987).

39. D. C. Hendry and D. Schuetzler, J. Am. Chem. Soc., 97, 7123 (1975).

40. E. C. Ashby and J. Oswald, J. Org. Chem., 53, 6068 (1988).

41. H. M. Walborsky and C. Zimmermann, J. Am. Chem. Soc., 114, 4996 (1992).

42. H. K. Kim and K. Matyjaszewski, Polym. Bull., 22, 441 (1989).

43. W. G. Boberski and A. C. Allred, J. Organomet. Chem., 88, 73 (1975).

44. J. A. Kerr, in "Free Radicals," Vol. I, J. K. Kochi, Ed., John Wiley \& Sons, New York, 1973, Chapter 1.

45. J. A. Hawari, D. Griller, W. P. Weber, and P. P. J. Gaspart, Organomet. Chem., 326, 335 (1987).

46. G. Raabe and J. Michl, Chem. Rev., 85, 419 (1985).

47. P. Trefonas, III, R. West, and R. D. Miller, J. Am. Chem. Soc., 107, 2737 (1985).

48. R. D. Miller, in "Silicon-Based Polymer Science: A Comprehensive Resource," Advances in Chemistry Series 224, J. M. Zeigler and F. W. G. Fearon, Ed., American Chemical Society, Washington, DC, 1990,
Chapter 24.

49. T. Karatsu, R. D. Miller, R. Sooriyakumaran, and J. Michl, J. Am. Chem. Soc., 111, 1140 (1989).

50. L. J. Mathias and C. E. Carraker, Jr., "Crown Ethers and Phase Transfer Catalysts in Polymer Science and Technology," Vol. 24, Plenum, New York, 1984.

51. S. Boileau, B. Kaempf, J. M. Lehn, and F. Schue, $J$. Polym. Sci., Polym. Lett. Ed., 12, 203 (1974).

52. S. Boileau, B. Kaempf, S. Raynal, J. Locoste, and F. Schue, J. Polym. Sci., Polym. Lett. Ed., 12, 211 (1974).

53. K. Matyjaszewski, J. Inorg. Organomet. Polym., 1, 463 (1991).

54. K. E. Ruehl, M. E. Davis, and K. Matyjaszewski, Organometallics, 11, 788 (1992).

55. (a) A. M. Basedos and K. H. Ebert, Adv. Polym. Sci., 22, 83 (1977), (b) W. B. Smith and H. W. Temple, J. Phys. Chem., 72, 4613 (1968), (c) A. M. Basedos and K. H. Ebert, Makromol. Chem., 176, 745 (1975).

56. R. D. Miller and D. Thompson, Polym. Prepr., 31, 300 (1990).

57. S. Gauthier and D. J. Worsfold, in "Silicon-Based Polymer Science: A Comprehensive Resource," Advances in Chemistry Series 224, J. M. Zeigler and F. N. G. Fearon, Ed., American Chemical Society, Washington, DC, 1990, Chapter 18. 